\title{
Immigrazione, Religione e Integrazione. Il caso delle comunità sikh e filippine in Lombardia*
}

\author{
di Samuele Davide Molli ${ }^{\circ}$ Maurizio Ambrosini ${ }^{\wedge}$
}

\section{Riassunto}

La trasformazione multireligiosa dell'Italia è uno dei più rilevanti fenomeni sociali seguiti ai flussi migratori, nonché una delle questioni più controverse. L'articolo entra nel merito del nuovo pluralismo e discute il rapporto tra le comunità religiose ristabilite dagli immigrati e i processi di integrazione che ne derivano. Dopo una rassegna della letteratura internazionale sul tema, il contributo prende in esame due casi studio rappresentativi dei tipi di pluralismo religioso: il caso dei sikh in provincia di Bergamo e dei cattolici filippini nella città di Milano; in chiave comparativa, si confronta il ruolo della religione per due significative esperienze di radicamento territoriale e di partecipazione economica degli immigrati in Lombardia. Nello specifico, in primo luogo, vengono ricostruiti i processi alla base dello sviluppo di una nuova geografia religiosa; si analizzano le differenti risorse che la partecipazione ai luoghi di culto fornisce ai fedeli sikh e filippini per fronteggiare le difficoltà derivanti dall'inserimento in nuovo contesto. Vengono poi esaminate le forme di accettazione nei confronti di tali tipi di pluralismo, e infine viene indagato lo spirito civico sviluppato invece verso la più ampia realtà sociale. L'articolo conclude mostrando come le comunità religiose, nonostante le difficoltà rilevate rispetto al loro riconoscimento, siano una risorsa per i processi di integrazione degli immigrati, con importanti ricadute per la società ricevente.

Parole Chiave: Immigrazione; Religione; Integrazione; Pluralismo Religioso; Cattolicesimo; Sikhismo.

\footnotetext{
* Gli autori desiderano ringraziare Elena Uccheddu per aver discusso e condiviso il suo lavoro di ricerca condotto con i lavoratori sikh impiegati nel settore zootecnico nella provincia di Bergamo; il suo contributo è stato di fondamentale importanza per la stesura del paragrafo dedicato ai bergamini della Bassa Bergamasca.

'Università degli Studi di Milano Statale. Corresponding author: Samuele.Molli@unimi.it.

${ }^{\wedge}$ Università degli Studi di Milano Statale.
}

Educational Reflective Practices (ISSNe 2279-9605), 1/2021 Special Issue Doi: 10.3280/erp1-special-2021oa12470 


\title{
Immigration, Religion and Integration. The case of Sikh and Fili- pino communities in Lombardy
}

\begin{abstract}
The multireligious transformation of Italy is one of the most important social phenomena following migration flows, as well as one of the most controversial issues. The article elaborates on the new pluralism and discusses the relationship between religious communities re-established by immigrants and the deriving integration processes. After a review of the international literature on the subject, the paper examines two case studies, representative of the types of religious pluralism: the case of the Sikhs in the province of Bergamo and of Filipino Catholics in the city of Milan; it works in a comparative key, and the role of religion is thus analysed for two significant experiences in terms of territorial rootage and economic participation of immigrants in Lombardy. Specifically, in the first place, the processes underlying the development of a new religious geography are reconstructed. Secondly, the different resources that religious participation provides to the Sikh and Filipino faithful are detailed; a series of helps they use in order to face the difficulties deriving from the settlement in a new context. The forms of acceptance towards these types of pluralism are then examined, and finally the civic spirit that these realities have instead developed towards the wider social reality is investigated. The article concludes by showing how religious communities, despite the difficulties encountered about their recognition, are a resource for the integration processes of immigrants, with important social repercussions also for the receiving society.
\end{abstract}

Keywords: Immigration; Religion; Integration; Religious Pluralism; Catholicism; Sikhism.

First submission: 03/07/2021, accepted: 31/08/2021

Available online: $30 / 09 / 2021$

\section{Introduzione}

I flussi migratori rappresentano il principale fattore di cambiamento del panorama religioso italiano. Nel corso degli ultimi tre decenni, l'insediamento di immigrati di differenti nazionalità ha infatti contribuito allo sviluppo e al consolidamento di un inedito pluralismo religioso (Pace, 2013; Ambrosini, Naso, \& Paravati, 2018). Come in molti altri Paesi europei, anche in Italia possiamo quindi osservare come la crescente diversità in ambito 
religioso sia uno dei fenomeni socioculturali più significativi seguiti ai processi migratori.

Questa transizione rappresenta però uno dei temi più controversi nel rapporto tra società italiana e popolazione immigrata, soprattutto per quanto riguarda i processi di integrazione. Più in generale, come ricostruito da J. Casanova (2007), la progressiva secolarizzazione delle società europee ha reso l'incorporazione delle religioni immigrate nella sfera pubblica motivo di tensioni. Le richieste in merito all'erezione di nuovi luoghi di culto e la rievocazione di identità ed appartenenze religiose prima sconosciute hanno infatti suscitato apprensioni e diffidenze in termini di sicurezza, convivenza e coesione sociale; inquietudini che trovano nell'Islam il principale riferimento e che spesso finiscono per convergere nella popolare tesi dello scontro di civiltà teorizzata da Huntington. Rispetto a questa visione, almeno due considerazioni vanno però discusse per inquadrare ed analizzare con più precisione il fenomeno.

In primo luogo, dai dati attualmente disponibili (nello specifico: Ismu, 2019; Idos-Confronti, 2020) - al netto dei limiti metodologici a cui è soggetto il calcolo delle stime - è possibile ricavare un quadro più preciso della nuova geografia religiosa promossa dagli immigrati. Tra gli stranieri residenti, se i fedeli musulmani sono circa un terzo del totale (33\% - 1.700.000), quelli cristiani rappresentano oggi la maggioranza $(52 \%-2.750 .000)$, a loro volta ripartiti nella componente ortodossa $(29 \%-1.530 .000)$, cattolica $(18 \%$ - 950.000) e protestante (4.5\% -235.000). Seppur numericamente più esigue, altre significative presenze comprendono i fedeli buddisti (125.000), induisti (163.000) e sikh (50.000).

Rispetto a questi dati, la diversità religiosa non è riconducibile e sovrapponibile alla sola presenza islamica: tra $i$ fedeli immigrati, la componente maggioritaria è quella cristiana. Osserviamo infatti una pluralizzazione endogena alle denominazioni cristiane storicamente insediate, come nel caso dei cattolici, dei protestanti - comprese alcune correnti pentecostali - così come dei Testimoni di Geova. Una diversificazione che interessa anche le religioni orientali, come nel caso del buddismo e dell'induismo: oltre ai praticanti autoctoni - che hanno contributo all'importazione di alcune correnti e tradizioni - sono aumentate le comunità etniche sorte in seguito all'arrivo di immigrati. Anche l'Islam presenta al proprio interno differenti componenti nazionali: metà dei fedeli proviene dal Nord-Africa (Marocco, Tunisia, Egitto), mentre troviamo una significativa presenza senegalese, una componente europea (Albania), e infine, più recentemente, si rileva anche la crescita di fedeli provenienti dall'Asia: come nel caso del Pakistan e del Bangladesh. 
Preso atto della composizione multi-sfaccettata del nuovo pluralismo, mutuando un concetto diffuso nell'ambito degli studi sul multiculturalismo, è possibile osservare come in Italia si sia di fatto consolidata una "superdiversità religiosa" (Pace, 2018). Se da un lato la società è diventata religiosamente plurale con il progressivo arrivo di nuove fedi, dall'altro si osserva anche una crescente differenziazione interna alle stesse confessioni, un processo rilevabile sia per le religioni storicamente insediate, come nel caso emblematico del cattolicesimo, sia per quelle più recentemente importate, ad esempio le differenti tradizioni e forme spirituali orientali; inoltre, le seconde generazioni stanno contribuendo a rendere maggiormente articolato il quadro presentato (Ricucci, 2017) per via di una più complessa intersezione tra provenienza, nazionalità e appartenenza religiosa.

Rispetto alla sempre più diversificata composizione del nuovo panorama religioso italiano, differente dalle comuni rappresentazioni che tendono invece a focalizzare l'attenzione solo sull'Islam, una seconda considerazione chiama in causa i vissuti e le pratiche religiose degli immigrati. Va infatti notato che solo recentemente in Europa ed in Italia, a differenza del contesto nord-americano (si veda nel merito: Foner \& Alba, 2008), le esperienze e le attività promosse all'interno dei luoghi di culto sono diventate oggetto di interesse. Il ruolo delle comunità sta infatti emergendo come campo di indagine empirica, sia in merito alle funzioni che svolgono per gli immigrati nei processi di inserimento e stabilizzazione, sia per le più ampie ricadute sociali e civiche che la stessa religiosità comporta per i contesti di ricezione (Ambrosini, Bonizzoni, \& Molli, 2021a). Alcuni studi osservano che la religione, una volta trapiantata, tende ad assumere nuovi significati, rispondendo ai bisogni che i migranti affrontano nel processo di insediamento. Fornisce un'opportunità di socialità e partecipazione, nonché uno spazio di attivismo per promuovere azioni e servizi solidali (Ambrosini, Bonizzoni, \& Molli, 2021b).

Oltre al piano descrittivo del pluralismo, il presente articolo intende infatti sviluppare una prospettiva analitica volta ad esaminare le differenti implicazioni correlate all'insediamento delle comunità religiose immigrate in nuovo contesto. Nello specifico, dopo una discussione rispetto alle più recenti correnti teoriche sul ruolo della religione in migrazione, l'articolo entra nel merito dei significati e delle pratiche osservati nei luoghi di culto ristabiliti. Da un punto di vista empirico, la comunità cattolica filippina e quella dei sikh costituiscono l'oggetto dell'indagine. La ricerca ha voluto prendere in esame due differenti gruppi di immigrati radicati nel territorio, che nel corso del tempo hanno promosso due importanti esperienze comunitarie a base religiosa, contribuendo in modo significativo al pluralismo in Lombardia: da 
un lato, importando nuove fedi, dall'altro diversificando in chiave etnica la religione storicamente maggioritaria.

Inoltre, i casi studio considerati rappresentano due esemplari forme di partecipazione economica degli immigrati al mercato del lavoro (definite in letteratura come specializzazioni etniche: Ambrosini, 2020); entrambe esperienze altamente rappresentative delle ragioni alla base della domanda di manodopera immigrata nel nostro paese. Un fenomeno, il primo, osservato in una zona agro-zootecnica - la Bassa Bergamasca - fuori dai centri cittadini, il secondo invece pertinente all'economia tipica dei contesti urbani, nello specifico Milano. Da un lato, un ambito occupazionale prettamente maschile, dall'altro invece femminile. La comparazione tra due storie migratorie quasi agli "antipodi" in termini sociologici, per genere, tipo di lavoro, zona di insediamento territoriale e soprattutto per confessione, permetterà quindi di avanzare nella comprensione del ruolo svolto dalla religione nelle rispettive esperienze di inserimento a livello locale, nonché di esaminare le implicazioni di tale religiosità anche per quanto riguarda i processi di integrazione nella società italiana.

\section{Le comunità religiose in diaspora: resilienza e trasformazione}

Nella letteratura europea ed italiana sul pluralismo religioso, una delle prospettive analitiche più seguite e discusse prende in considerazione l'intreccio tra la dimensione politica, normativa ed istituzionale (si veda per esempio: Berger, Davie, \& Fokas, 2010). In particolare, la giurisprudenza prodotta in merito al riconoscimento delle nuove confessioni è uno dei principali ambiti di interesse (per il caso italiano: Ferrari, 2012). È possibile inquadrare e riassumere questa prospettiva come un approccio di marca istituzionalista. Discute gli aspetti giuridici, i modelli di governance, la storia delle relazioni tra Stato e religioni, le differenti traiettorie dei processi di secolarizzazione, nonché le culture e le tradizioni politiche all'interno delle quali il nuovo pluralismo religioso promosso dall'arrivo di immigrati trova forme di regolazione (Triandafyllidou \& Magazzini, 2020).

Oltre a questo ambito, più recentemente, anche un interesse di tipo sociologico si sta progressivamente sviluppando ed affermando; una prospettiva di ricerca volta ad approfondire il pluralismo "dal basso" e "nel merito". Un approccio che prende in considerazione, oltre il contesto istituzionale, le comunità e i suoi fedeli. In altri termini, oggetto di analisi sono i processi tramite i quali le nuove realtà religiose si sono sviluppate, organizzate e strutturate a livello locale; si considerano i vissuti dei fedeli che vi partecipano, $i$ vari significati dell'appartenenza religiosa, $i$ tipi di relazioni instaurate nelle 
comunità, le forme di cooperazione, le attività promosse all'interno e verso l'esterno, i contatti con le istituzioni locali, nonché le leadership che possono emergere e i legami transnazionali con la madrepatria.

In questo senso, la letteratura internazionale, soprattutto nord-americana (si veda Cadge \& Ecklund, 2007), fornisce una serie di evidenze nel merito, raramente assunte nel dibattito italiano. La lunga storia delle migrazioni negli Stati Uniti fornisce infatti un riferimento empirico per approfondire il ruolo delle religioni nei processi di insediamento (Hirschman, 2004), come per il caso degli emigranti italiani nel secolo scorso (Vecoli, 1969; Sanfilippo, 2007), quando le parrocchie etniche risposero ai bisogni e alle necessità dei nostri connazionali, offrendo anche protezione rispetto alle discriminazioni subite Una letteratura che fatica però oggi ad emergere rispetto all'Italia come Paese di immigrazione.

In primo luogo, gli spazi religiosi ristabiliti all'estero emergono come contesti dove rievocare forme di appartenenza culturale ed etnica (Min, 2010) e nei quali poter trovare riconoscimento e familiarità rispetto all'esperienza dello sradicamento. La religione diventa "una catena di memoria" (Hervieu-Leger, 2000) che permette di mantenere vivo il legame con la madrepatria (Orsi, 2010). In questo senso, cultura, etnia e religione sono elementi profondamente intrecciati, e vengono ri-materializzati dagli immigrati (Tweed, 1997) che stanno trasformando luoghi periferici ed ex industriali, antiche chiese e santuari, in nuovi spazi dove importare immagini, statue, simboli e simulacri, e dove riprodurre processioni e feste nazionali. Questa "geografia della memoria" è oggi sempre più re-interpretata ed analizzata nella prospettiva teorica del transnazionalismo (Levitt, 2007), uno dei più recenti approcci negli studi migratori. I luoghi religiosi sono diventati infatti tra i più importanti snodi locali dove ristabilire e sviluppare canali di connessione sovranazionali, di tipo emozionale, culturale e sociale, tra madrepatria e società ricevente.

Oltre l'aspetto della continuità culturale, l'esperienza religiosa assume anche forti connotati relazionali (Ebaugh \& Curry, 2000), soprattutto in termini di capitale sociale che deriva dalla partecipazione (Foley \& Hoge, 2007; Molli, 2020). Nelle comunità si creano infatti significative opportunità per sviluppare legami e conoscenze in un nuovo contesto. Le relazioni instaurate hanno poi importanti implicazioni emotive e psicologiche (Gozdiack, 2002; McMichael, 2002; Dorais, 2007). Gli incontri e i ritrovi religiosi aiutano le persone nel condividere le difficoltà affrontate quotidianamente, un appuntamento particolarmente significativo per coloro che si trovano in condizioni legali precarie (Menjívar, 2006). Per esempio, Connor (2012), in uno studio quantitativo ha individuato forti connessioni tra il benessere dei migranti e la loro partecipazione religiosa. La combinazione tra familiarità culturale e 
capitale sociale può essere quindi ridefinita come una risorsa per la "resilienza" degli immigrati nei processi di insediamento.

In secondo luogo, le istituzioni religiose, quando trapiantate in nuovo contesto, tendono a promuovere nuove funzioni sociali. Le comunità diventano punti di riferimento dove i migranti cercano vari tipi di aiuto (Ley, 2008). Valori quali empatia ed accoglienza, trasversali a molte confessioni, sono un fattore di facilitazione per forme di cooperazione e reciprocità volte a rispondere ai bisogni e alle criticità che inevitabilmente accompagnano gli immigrati anche nelle comunità religiose. Questo attivismo solidale diventa un motivo centrale del loro insediamento all'estero (Guest, 2003; Mooney, 2009; Stepick, Rey, \& Mahler, 2009). È possibile definire questo ampliamento dei servizi, in termini di supporti, forme di aiuto ed orientamento, come un "welfare dal basso" (Ambrosini, Bonizzoni, \& Molli, 2021b) che sostiene e rafforza la cittadinanza sociale degli immigrati.

Insieme all'attivismo solidale, altre trasformazioni possono occorrere; quando una comunità ristabilisce un nuovo corso, il background socio-istituzionale precedente viene a mancare e il suo funzionamento affronta nuove sfide. In questo senso, il dislocamento produce anche trasformazione (Yang \& Ebaugh, 2001). Per esempio, nei modelli organizzativi si osserva spesso un maggiore protagonismo dei laici (Warner, 2000) in ragione del fatto che le comunità si ristrutturano spesso ex novo grazie al loro protagonismo. Tale processo promuove nuovi ruoli, e nuove forme di leadership, con importanti ricadute anche in termini di autostima, dignità, responsabilità, oltre che di rispettabilità verso l'esterno; significative opportunità per rispondere alla mobilità sociale verso il basso sperimentata da molti immigrati in un nuovo contesto. Anche il ruolo delle donne all'interno delle comunità può essere ambito di rinegoziazione (Bonifacio \& Angeles, 2010). Mentre la religione in migrazione è spesso associata alla riproduzione di gerarchie e di dominio patriarcale, in realtà i modelli di genere possono invece mutare nelle religioni trapiantate (Nyhagen \& Halsaa, 2016), producendo un'interessante tensione tra conservatorismo e cambiamento, compresa l'affermazione di nuove leadership femminili (Ebaugh \& Chafetz, 1999).

Infine, le seconde generazioni sono uno degli elementi di trasformazione più significativi per le fedi in migrazione (Ricucci, 2017); rispetto alle esigenze dei genitori, i fedeli più giovani cercano differenti forme di coinvolgimento religioso, esprimendo nuovi bisogni e desideri; processi che possono portare a tensioni e scissioni, così come alla progressiva promozione di attività socio-religiose e di leadership dal nuovo profilo.

Se resilienza e trasformazione caratterizzano l'esperienza delle comunità religiose dei migranti, fornendo importanti risorse per l'inclusione dei fedeli, anche nodi critici possono però svilupparsi. La socialità faticosamente 
ristabilita, nel produrre un forte senso di appartenenza, può trasformarsi in un eccesso di aspettative, ossia pressioni al conformismo rispetto ai comportamenti dei membri. Troviamo inoltre che la coesione interna può portare a segnare un netto confine verso l'ambiente esterno, percepito come minaccioso e pericoloso per i valori e per la morale riprodotta internamente. Una forma di coinvolgimento di tipo reattivo, che porta ad un ripiegamento delle comunità su sé stesse, limitando in tal modo lo scambio e i contatti sociali con altre istituzioni locali.

Questa serie di riferimenti, tra resilienza, trasformazione e criticità, verrà quindi considerata rispetto ai casi studio e alle evidenze raccolte, al fine di comprendere quale ruolo ricopre la religione nei processi di inserimento ed integrazione dei sikh e dei filippini cattolici in Lombardia.

\section{La presenza demo-religiosa dei sikh e dei filippini in Lombardia}

La possibilità di censire la presenza di fedeli sikh in Italia non è priva di difficoltà (Omenetto, 2015): sono infatti differenti le fonti statistiche, e in parte discordanti. Secondo il centro studi Cesnur ${ }^{1}$, i sikh indiani sono circa 70.000 , la seconda comunità in Europa dopo quella presente in Gran Bretagna (400.000). Stando invece alle elaborazioni fornite dal rapporto CaritasMigrantes (2020) sono circa 51.000, una stima simile a quella fornita da Ismu (2019); secondo Bertolani (2013) i fedeli sikh sono invece circa 85.000, mentre per Gallo (2012) sono più di 100.000.

Tali discrepanze sono dovute, in primo luogo, alla metodologia con la quale sono elaborate le stime, che solitamente, in termini statistici, ripondera per le comunità di immigrati presenti in Italia le appartenenze religiose osservate nei paesi di origine. Nel caso del sikhismo le difficoltà sono però dovute alla capacità di stimare in modo effettivo la sua distribuzione tra gli immigrati che provengono dall'India. Nello specifico, in Italia la grande maggioranza degli indiani proviene dalla regione del Punjab, dove, a differenza del resto del Paese, la popolazione è in maggioranza sikh. Bertolani (2013), sulla base di differenti lavori di ricerca condotti sul tema, quantifica tale proporzione in circa il $70 \%$. Infine, va tenuto in considerazione che nel calcolo delle stime non sono compresi gli stranieri in condizione irregolare o coloro che non sono ufficialmente iscritti ad un'anagrafe comunale, né coloro che hanno acquisito la cittadinanza italiana. Stando a queste osservazioni, gli indiani presenti in Italia ad inizio 2020 sono circa 160.000 (Idos-

\footnotetext{
${ }^{1} \mathrm{Si}$ veda la sezione liberamente accessibile online: https://cesnur.com/gruppi-di-originesikh/la-religione-sikh/.
} 
Confronti, 2020), su poco più di 5 milioni di stranieri ufficialmente residenti (circa il 3,0\%); mentre in Lombardia, sono circa 48.000, il 4,0\% del totale degli stranieri in regione (circa 1.150.000), nonché un terzo degli indiani di tutta Italia. Tra questi, possiamo quindi supporre che si trovi anche una significativa quota proveniente dall'area del Punjab; una presenza che ha contributo in modo significativo all'insediamento del sikhismo in regione.

Il secondo dato da considerare concerne infatti l'erezione di luoghi di culto da parte dei fedeli sikh, comunemente identificati come Gurdwara ${ }^{2}$. Sulla base della ricerca che abbiamo condotto in Lombardia è possibile rilevare la presenza di 17 templi; di questi, dieci si trovano tra le province di Bergamo (3), Brescia (4) e Mantova (3). Molti dei Gurdwara individuati si concentrano infatti in aree vocate ad attività agro-pastorali, come nel caso in esame della Bassa Bergamasca, situata a sud della città di Bergamo e confinante con Brescia e Cremona. La concentrazione di questi luoghi di culto si correla al fatto che nel corso del tempo una quota molto significativa di immigrati sikh si è progressivamente inserita nel mercato del lavoro locale, formando una nicchia occupazionale etnica. Nello specifico, gli immigrati indiani sono impiegati come mungitori presso le aziende agricole situate in molte cascine della "bassa".

Sono state quindi raccolte 20 interviste semi-strutturate - svolte da ottobre 2019 a gennaio 2020 - con lavoratori sikh impiegati nel settore zootecnico, uomini compresi tra i 22 e i 53 anni - che nel fine settimana frequentano le comunità religiose. Le interviste condotte hanno infatti approfondito sia la condizione lavorativa (il tipo di impiego e la conciliazione vita-lavoro), sia i significati e le motivazioni alla base della partecipazione religiosa.

Per quanto riguarda la presenza di immigrati filippini, va in primo luogo osservato che sono circa 170 mila in Italia (il 3\% degli stranieri), in Lombardia sono invece circa 60 mila (il 5\% della popolazione immigrata residente). In regione si concentra quindi più di un terzo degli immigrati filippini residenti in Italia; dati analoghi al caso dei sikh. In merito alla loro appartenenza religiosa, il cattolicesimo è largamente maggioritario (più dell' $80 \%$ ). $\mathrm{Nel}$ corso del tempo sono molte le comunità cattoliche a base etnica che si sono sviluppate; il loro insediamento rappresenta di fatto uno dei più significativi vettori di trasformazione del cattolicesimo lombardo in chiave multiculturale.

In questo senso, osserviamo come in regione la Chiesa cattolica abbia progressivamente assunto un profilo sempre più multietnico. Secondo le stime disponibili in Lombardia risiedono infatti circa 275 mila immigrati

\footnotetext{
${ }^{2}$ Si rimanda al lavoro svolto da Barbara Bertolani (2013) dove è possibile trovare un'introduzione al Gurdwara come luogo di culto per i sikh e alle sue principali caratteristiche.
} 
cattolici (si veda Ismu, 2019), la più alta concentrazione in Italia, quasi un quarto della popolazione straniera lombarda. Questo progressivo insediamento ha prodotto a sua volta una complessa e assai diversificata geografia del cattolicesimo migrante. In molte delle città capoluogo della regione sono nate svariate "chiese etniche" (più di 80 ), ossia comunità religiose di immigrati cattolici che hanno trovato sede in una chiesa locale; nel caso dei filippini, secondo la ricerca condotta, tra le 16 individuate, Milano rappresenta l'epicentro (11), alla quale seguono Bergamo (1), Brescia (1), Mantova (1) e Como (1). La presenza di luoghi di culto, come nel caso dei sikh, rispecchia infatti l'insediamento della popolazione immigrata; in particolare, a Milano città la comunità di immigrati numericamente maggioritaria è quella filippina (circa il 15\% degli stranieri residenti); inoltre, 1'85\% dei filippini presenti in regione risiede proprio nella provincia milanese. Nel corso del tempo, il mercato del lavoro metropolitano, nello specifico il settore dei servizi alla persona e alla casa, è stato un polo occupazionale attrattivo per gli immigrati filippini, soprattutto per la componente femminile (il $60 \%$ circa della comunità milanese), come nel caso di colf, baby-sitter, addette alle pulizie, custodi, e lavoratrici in vari ambiti di assistenza alle famiglie italiane. Sono state quindi condotte 20 interviste semi-strutturate - svolte nel 2019 e nel corso del 2020 - con donne che partecipano attivamente alle chiese etniche, a cui si aggiungono numerose note di osservazione raccolte presso gli stessi luoghi di culto e altre 5 interviste con i sacerdoti che seguono le stesse comunità.

Grazie alla triangolazione tra differenti tipi di dati qualitativi raccolti, entreremo ora nel merito delle esperienze religiose dei fedeli immigrati, considerando i processi che hanno portato all'insediamento delle comunità nel territorio, i significati e le attività sociali promosse all'interno e le forme di riconoscimento in merito alla loro presenza rilevabili a livello locale.

\section{Una nuova geografia religiosa: l'in-visibilità di templi e santuari}

Dopo aver definito la presenza demo-religiosa dei sikh e dei filippini, il primo ambito di indagine prende in considerazione i luoghi di culto sorti in seguito al loro progressivo insediamento. La creazione di un tempio, così come la concessione di una chiesa ad un gruppo di fedeli stranieri, è infatti uno dei segni più visibili e tangibili della presenza di una comunità di immigrati; una delle principali modalità per ricostruire ed affermare una nuova identità sul territorio. Da questo punto di vista, la geografia religiosa è di particolare interesse poiché chiama in causa tre riferimenti nel caso della popolazione immigrata: la madrepatria, la comunità ristabilita e la società 
ricevente. Di fatto, in questi spazi si intrecciano a vario titolo tre livelli di analisi: transnazionale, nazionale e locale.

Prendendo in esame la geografia religiosa dei sikh osserviamo che molti dei siti individuati non sono immobili costruiti $a b$ origine per la pratica religiosa, bensì sono edifici già esistenti; si tratta di fabbriche, officine, magazzini e depositi non più adibiti alla loro originaria funzione, e situati in zone industriali di piccoli comuni che compongono la geografia della Bassa Bergamasca. I sikh hanno quindi avuto in uso, e spesso riqualificato, spazi abbandonati o dismessi; anche se non si osservano particolari cambiamenti urbanistici visibili dall'esterno, i luoghi sono però trasformati all'interno e dotati di un nuovo significato religioso.

Per esempio, a Cortenuova, un piccolo comune di duemila abitanti, il tempio si trova nell'area industriale; a Covo, una municipalità con poco più di quattromila abitanti, il centro sikh è stato ricavato in un'area periferica ad uso commerciale. Sebbene indicazioni, cartelli e targhe possano indicarne la presenza, di fatto gli spazi non sono immediatamente riconoscibili, essendo incorporati in piccoli distretti manifatturieri o commerciali situati in snodi di intersezione che collegano e servono le aree agro-zootecniche circostanti.

Anche a Borgo san Giacomo, in provincia di Brescia, a pochi chilometri dalla Bassa Bergamasca e frequentato per vicinanza da molti dei sikh intervistati, il tempio si trova in un'area industriale molto estesa, formata da un complesso di capannoni, alcuni dei quali in uso alla stessa comunità sikh per proprie attività commerciali (es. markets). Un processo analogo è osservabile anche a Mantova, a Castellucchio, un paese di cinquemila abitanti, con caratteristiche simili alla Bassa Bergamasca per la diffusione di attività zootecniche, dove i sikh hanno ricavato un tempio da un capannone situato all'interno di un distretto industriale.

Possiamo quindi osservare almeno due processi; in primo luogo, grazie ai loro sforzi, i sikh hanno riconvertito edifici secolari in nuovi templi, che sono progressivamente diventati un riferimento per la riacquisizione di un'identità collettiva, attirando i fedeli che risiedono e lavorano in varie aree limitrofe. In secondo luogo, la creazione di un tempio ha delle implicazioni di più ampio respiro verso il contesto in cui si è insediato; nello specifico, una nuova confessione di fatto entra nella sfera pubblica della società ricevente. Va però osservata una certa ambivalenza rispetto a questo aspetto; l'insediamento di tali comunità religiose avviene nella modalità della riconversione, che assume un profilo di semi-(in)visibilità. In tal senso, da un lato il processo può essere interpretato come mimetico (Gallo, 2012), essendo tali templi insediati in aree industriali ove spesso si confondono. Dall'altro lato va però tenuto in considerazione il quadro giuridico-istituzionale che ne regola il riconoscimento, un complesso quadro normativo che limita e complica la 
possibilità di aprire ex novo (in realtà, anche di riconvertire) un edifico ad uso religioso. Infine, vanno valutati anche gli aspetti prettamente pragmatici, relativi all'opportunità di reperire ed affittare (o comprare) con più facilità uno spazio commerciale, spesso dismesso o sfitto.

Per il caso dei filippini, analizzando la geolocalizzazione delle chiese dove le comunità cattoliche etniche hanno trovato sede, emerge che il loro insediamento si è sviluppato soprattutto in aree centrali e in chiese storiche della città di Milano. In questo senso, i fedeli immigrati hanno trasformato in spazi animati chiese locali ormai povere di fedeli e scarsamente utilizzate. In Milano, così come in molte delle città capoluogo lombarde e italiane, le parrocchie dei centri città hanno spesso un numero elevato di chiese; alcune di queste sono oggi poco frequentate, e talvolta abbandonate. La disponibilità di questi spazi facilita dunque la possibilità di concederli in utilizzo ad un gruppo di fedeli immigrati che richiede la possibilità di poterli avere in uso per ricreare la propria comunità etnico-religiosa. Nei fine settimana, in accordo agli impegni lavorativi, possiamo infatti osservare una specifica forma di mobilità: i cattolici filippini, dalla provincia e dalla periferia dove risiedono, si spostano per partecipare e frequentare la chiesa etnica sentita come familiare. Anche nei casi in cui le comunità non abbiano sede in chiese così centrali, troviamo comunque edifici religiosi di minor interesse per i fedeli autoctoni -come piccole cappelle, tempietti o santuari- tipicamente sottoutilizzati dalla parrocchia di riferimento.

In tal senso, i filippini, se da un lato, in quanto cattolici, a differenza di altre confessioni religiose (e dei sikh) non devono affrontare il problema di trovare uno spazio -e soprattutto uno spazio con una destinazione d'uso religiosa- dall'altro lato va invece notato che si tratta spesso di luoghi di "secondo ordine", nonostante siano ubicati in aree centrali e semi-centrali delle città. Le chiese assegnate sono tipicamente edifici piccoli, antichi e poco attrezzati per le differenti attività interne. Il culto è infatti solo uno degli appuntamenti promossi, seguito o preceduto da una serie di eventi o ritrovi sociali. Inoltre, le chiese non sono sempre in concessione esclusiva, ma nella maggior parte dei casi prestate e condivise con la parrocchia italiana; in altri, le comunità filippine usano anche più chiese a rotazione sulla base delle disponibilità, un aspetto che limita ancor di più le possibilità di uso del luogo di culto rispetto alle esigenze di socialità dei fedeli.

Gli immigrati stranieri, da questo punto di vista, usano spazi, ma sempre negoziati e prestati da un'istituzione della società maggioritaria, su base discrezionale delle parrocchie locali; di fatto, nella maggior parte dei casi, sono ospiti. Infine, la visibilità della presenza di una comunità filippina è esternamente celata da un processo mimetico: gli edifici religiosi sono infatti gli stessi usati dai fedeli della religione maggioritaria nel Paese. 
In conclusione, possiamo quindi osservare come la creazione di nuove comunità religiose abbia trasformato e diversificato la geografia urbana locale; un processo che avviene tramite una riconversione di uno spazio, sacro o secolare, già esistente. In questo senso, l'analisi di tale geografia rivela come il fenomeno sia però spesso in-visibile, presente ma non sempre direttamente percepibile; le comunità hanno infatti dovuto negoziare il loro insediamento attraverso processi mimetici. I templi sikh si sono sviluppati in aree industriali e commerciali, le chiese etniche trovano invece posto nei centri storici delle città confondendosi nelle parrocchie locali. Nonostante queste forme di negoziazione, nuove identità religiose e culturali si sono comunque consolidate, ed entrano a far parte dello spazio pubblico.

Procederemo ora nell'analisi di questa geografia prendendo in esame i significati che questi luoghi ricoprono per i fedeli che vi partecipano, incluse le differenti attività che si sono sviluppate internamente.

\section{Dalla stalla al tempio: l'esperienza religiosa dei bergamini sikh}

Come ricordato in precedenza, gli immigrati sikh nell'area della Bassa Bergamasca sono in maggioranza occupati nel settore zootecnico. Nello specifico, ricoprono il ruolo di "bergamino", ossia colui che è addetto all'allevamento del bestiame e alla mungitura, nonché a tutte quelle attività inerenti alla cura della stalla. Mentre negli ultimi decenni la mansione del "bergamino" ha perso capacità di attrazione tra la manodopera locale, la sua necessità non è però diminuita. Gli immigrati dal Punjab hanno dunque risposto ad una sfasatura tra domanda e offerta in uno specifico settore del mercato del lavoro, e il loro progressivo impiego ha portato al consolidamento di una nicchia occupazionale etnica. Nel corso del tempo, le reti tra connazionali hanno agevolato la circolazione di informazioni e referenze, e hanno rafforzato l'immagine dei sikh in termini di affidabilità tra gli imprenditori locali; acquisita fiducia, sono quindi diventati il principale bacino di riferimento per la ricerca di bergamini nella Bassa Bergamasca.

Quella del mungitore è però un'attività faticosa ed impegnativa, che richiede almeno due turni di lavoro giornalieri, mattutino e pomeridiano (in quanto la mungitura deve essere effettuata ogni dodici ore), e di fatto prevede la presenza in stalla dello stesso lavoratore per quasi tutto l'arco della giornata. Se da un lato, nelle interviste condotte, quello del bergamino è un impiego accettato dai sikh poiché ben retribuito - un compenso che su scala

\footnotetext{
${ }^{3}$ Nella tradizione rurale della pianura padana il bergamino è una storica e tipica figura lavorativa presente nel sistema socio-economico della cascina (o corte colonica).
} 
transnazionale permette la possibilità di miglioramento del proprio status sociale e di quello della famiglia della madrepatria - dall'altro comporta però una serie di svantaggi. Una delle principali difficoltà emerse, oltre la fatica fisica, concerne la solitudine e la mancanza di tempi liberi. Inoltre, la vita del bergamino si concentra nella cascina, dove spesso, come appurato, alloggia. Questa soluzione, se da un lato permette di ovviare al problema della ricerca e del pagamento di un domicilio, dall'altro si traduce in una situazione di isolamento sociale.

Rispetto a tale forma di segregazione spaziale e relazionale, e ad una temporalità esistenziale strettamente dettata dagli impegni lavorativi, la possibilità di frequentare il tempio è un fattore importante per il benessere psicofisico dei bergamini.

Di seguito, alcune interviste raccolte nel corso del lavoro sul campo:

(...) Come tutti vado tutte le domeniche (al tempio). Vado con lo zio con cui vivo. Andiamo insieme a Covo o a Cortenuova. Ci sono tante persone, ho conosciuto tante persone frequentando il Gurdwara (Bergamino, 22 anni, in Italia da 3 anni).

Io ci vado tutti i giorni dopo il lavoro (al Gurdwara), anche solo per cinque minuti. La domenica è il giorno più importante, ma io ci vado tutti i giorni. Pregare è importante. Poi spesso c'è altra gente, non sono solo (Bergamino, 28 anni, in Italia da 8 anni)

Nel primo caso emerge come il tempio offra la possibilità di frequentare un contesto alternativo alla cascina, dove poter allacciare nuove conoscenze oltre i soli colleghi di lavoro, che per di più sono spesso familiari. Nel secondo, emerge come questa socialità sia importante per ovviare alla solitudine che inevitabilmente deriva dall'esperienza lavorativa nella cascina. Due osservazioni che ricorrono in molte delle interviste condotte con i bergamini:

Al Gurdwara ci vado tutte le domeniche mattina (...) Ogni domenica sono là con altra gente, preghi, passi del tempo con loro, parli del più e del meno. Anche in Italia voi uscite dalla chiesa e parlate con altre persone. Non è solo pregare la domenica, incontri anche gli amici che non vivono nel tuo stesso paese. Io ho tanti amici che non vivono qui (Bergamino, 51 anni, in Italia da 26 anni).

Si certo, io vado [tutte le] domeniche. Io porto [con me] mia moglie e figli. Andiamo e insieme preghiamo. Poi incontriamo [altri] amici, tutti la domenica andiamo. Tu preghi e stai bene poi vedi tanta gente e stai bene (Bergamino, 35 anni, in Italia da 17 anni). 
Per i sikh la frequentazione della propria comunità religiosa permette da un lato di creare nuove relazioni sociali, dall'altro di ricentrare le reti etniche geograficamente disperse nelle campagne della bassa dove i lavoratori, e i loro nuclei familiari se ricongiunti, risiedono senza particolari contatti esterni. Inoltre, come si può notare, la domenica, di fatto, diventa il giorno principale per frequentare il tempio, anche se per la religione sikh non è ufficialmente definita come tale nel calendario liturgico; per effetto di un sincretismo dettato dalle circostanze - gli altri connazionali, anche se non mungitori, sono occupati nella manifattura o nel commercio e il loro tempo libero si concentra nel fine settimana - il ritrovo comunitario domenicale si trasforma quindi in un appuntamento fisso.

Nei Gurdwara, gli immigrati sikh hanno quindi trovato uno spazio relazionale alternativo all' isolamento della cascina, ricavando anche un tempo differente da quello strettamente definito dalla mansione di bergamino. La religione è quindi un importante fattore di resilienza: il tempio, nel fornire $\mathrm{e}$ rievocare un ambiente culturale sentito come familiare, offre infatti un contesto ove trovare riconoscimento, costruire capitale sociale, e riacquistare energie funzionali al benessere personale. Inoltre, la frequentazione permette di liberarsi in questo modo dalla sola immagine di bergamino che accompagna i sikh durante la quotidianità settimanale.

\section{Dalla casa alla chiesa: oltre lo spazio domestico, il cattolicesimo delle colf filippine}

Il caso delle donne filippine impiegate come colf ha rilevanti analogie con quello degli immigrati sikh rispetto al fenomeno delle specializzazioni etniche nel mercato del lavoro. Le reti tra connazionali hanno infatti agevolato il progressivo consolidamento di una manodopera etnicamente definita per uno specifico tipo di mansione - spesso un'occupazione svalutata socialmente che non trova lavoratori(ci) locali. In questo senso, le donne filippine impiegate come colf nella città di Milano sono un caso emblematico di convergenza tra domanda e offerta di lavoro, tanto che, nel linguaggio parlato (ma non solo), l'identificazione tra la mansione di colf e il termine "filippina" è diventata un cliché.

$\mathrm{Nel} 2020$, tra gli 850 mila lavoratori registrati ufficialmente ${ }^{4}$ nel settore domestico, suddiviso in due principali tipi di rapporti di lavoro (colf ed

\footnotetext{
${ }^{4}$ Secondo ASSINDATCOLF, agli 850 mila domestici con contratto vanno però aggiunti circa 1,2 milione di lavoratori in nero; pur non avendo dati nel merito, possiamo comunque dedurre che una cospicua quota di questi rapporti irregolari coinvolga la popolazione immigrata. $\mathrm{Si}$
} 
assistenza familiare), il 70,3\% sono stranieri (INPS, 2020). Di questi, in Italia, 67.000 sono filippini (quasi 1' $8 \%$ dei lavoratori del settore), tra i quali circa 57.000 sono impiegati come colf (1'85\%), di cui le donne rappresentano una netta maggioranza. Possiamo quindi dedurre che molte siano concentrate proprio nella città di Milano; in provincia risiede infatti 1' $85 \%$ dei filippini residenti in Lombardia.

Nel merito dell'esperienza lavorativa, questa prevede normalmente tre tipi di mansioni: cucinare, pulire, curare (Lutz, 2011), compresa una serie di micro-attività inerenti alla gestione della casa. Rispetto alle implicazioni, oltre la fatica fisica e la noia derivante dalla ripetitività delle attività, il tipo di mansione è assai costrittivo per l'autonomia personale nel caso di coabitazione tra datore e dipendente; qualora invece si tratti di una collaborazione ad ore, l'impiego implica spesso lunghi viaggi, se non spostamenti giornalieri tra più datori. Il lavoro di colf si svolge per tutta la settimana in uno spazio limitato: un appartamento privato; inoltre, in termini relazionali, gli scambi sociali sono continuamente asimmetrici. In secondo luogo, subentra, direttamente o indirettamente, la domanda di coinvolgimento emotivo per la collaboratrice, essendo l'ambito di lavoro uno spazio familiare, in continuo contatto con bambini e anziani da seguire; per di più, non è rara la richiesta di ore aggiuntive che le stesse necessità della vita familiare dettano regolarmente.

Rispetto alle difficoltà riscontrate, la possibilità di frequentare la propria comunità religiosa è una significativa valvola di sfogo, un modo per trovare e costruire nuove relazioni oltre quelle (asimmetriche) lavorative, grazie alle quali condividere le difficoltà della quotidianità:

Ricordo quando sono arrivata ... i miei amici mi dicevano vieni qui, vieni in Chiesa, qui trovi una famiglia, e sinceramente ho trovato una nuova famiglia, se durante la settimana devi correre... qui almeno puoi essere libero, puoi parlare, puoi cantare e pregare nella tua lingua, puoi chiacchierare liberamente e le persone possono capire quello che vuoi dire... normalmente è difficile... quando arrivi devi imparare tutto, in poco tempo devi imparare la città, come muoverti, impari un nuovo lavoro, e quando ti fermi senti che le tue energie sono provate ... quindi ecco... la chiesa è come una famiglia ... (Rosalie, colf e fedele chiesa filippina).

Come per Rosalie, per molte delle donne incontrate, all'inizio, soprattutto se primo-migranti, la partecipazione diventa una preziosa opportunità per costruire relazioni sociali oltre gli impegni lavorativi, nonché un contesto dove problemi e difficoltà possono essere compresi poiché trasversali.

veda il comunicato ufficiale: https://assindatcolf.it/lavoro-nel-2020-saldo-positivo-per-effetto-sanatoria-nel-lavoro-domestico-ma-allarme-blocco-pratiche-emersione/. 
Abbiamo inoltre osservato che nelle chiese prese in considerazione la socialità a base religiosa ha portato alla creazione di differenti gruppi interni, che contribuiscono a fornire una serie di risorse. Questa complessa microorganizzazione offre infatti orientamento per varie esigenze, accoglienza attraverso la diffusione di informazioni per posti letto o richieste di lavoro, e sostegni per spese gravose ed impreviste. Forme di reciprocità e di mutualismo che emergono dal capitale sociale costruito internamente. I gruppi, oltre alle forme di aiuto, organizzano anche svariate attività, sia religiose sia conviviali, come feste, eventi, pranzi e pellegrinaggi, offrendo in tal modo molte opportunità per conciliare la propria appartenenza religiosa e culturale con il desiderio di socialità.

Questo attivismo produce a sua volta una serie di ruoli e responsabilità, con importanti ricadute per il benessere dei fedeli; come raccontato dalla stessa Rosalie:

Durante la settimana lavoro in una famiglia di Milano da tanti anni (...) ma qui in Chiesa coordino un gruppo, facciamo tante attività! All'inizio frequentavo e basta ... ma ha iniziato a piacermi, e piano piano sono diventata una responsabile...questo prevede molti più impegni, perché devi organizzare persone, e devi organizzare eventi, anche in accordo con gli altri gruppi (...) mi piace molto...queste sono soddisfazioni, mi sento coinvolta, mi piace...

Si può dunque notare come il coinvolgimento in questi gruppi, oltre a fornire una serie di servizi, permette anche di riabilitare l'immagine stereotipata che accompagna le donne filippine come colf; se da un lato la propria appartenenza nazionale è diventata un "vantaggio competitivo" in termini di inserimento lavorativo, dall'altro riproduce però (ed istituzionalizza) un'immagine subalterna e servizievole.

La partecipazione religiosa offre quindi importanti opportunità relazionali, agevola l'accesso ad aiuti ed orientamento, e offre una preziosa possibilità per reagire allo stigma che accompagna le donne filippine nella città di Milano.

\section{Accettazione e civismo delle comunità religiose nelle realtà locali}

In precedenza, abbiamo considerato come gli immigrati sikh e filippini abbiano negoziato, non senza difficoltà, la possibilità di ricreare spazi dove insediare le proprie comunità religiose; abbiamo quindi analizzato tali luoghi dal punto di vista dei significati che assume la religiosità nelle esperienze di vita dei migranti; prendiamo ora in considerazione il riconoscimento e il 
ruolo di queste realtà a livello locale. Nello specifico, se la creazione del luogo di culto sancisce l'entrata nella sfera pubblica di un'identità religiosa, dall'altro lato vanno però prese in considerazione le forme di riconoscimento e il tipo di relazioni che le comunità hanno instaurato nel contesto di ricezione.

Per i sikh abbiamo osservato come vari edifici secolari siano stati riconvertiti in spazi religiosi; un processo mimetico, non sempre percepibile, e dettato dalle circostanze. Nella maggior parte dei casi tale riconversione non ha suscitato particolari reazioni, ma in altri ha invece sollevato controversie. Per esempio, a Suzzara, in provincia di Mantova, la comunità sikh ha ricavato un tempio in un piccolo capannone, incontrando numerose difficoltà nell'acquisto e nell'ottenimento del cambio di destinazione d'uso dell'edifico; la municipalità ha infatti avviato una lunga e complicata interlocuzione. A Brescia, un tempio è stato allestito in un ex laboratorio situato in una zona industriale, ma abbastanza centrale, della città; rispetto alla questione della destinazione d'uso del locale sono emerse varie controversie e polemiche. Possiamo quindi osservare come la "geografia del riconoscimento" sia assai differente a livello locale, una variabilità che dipende da come l'insediamento di un tempio diventi una questione politica. In questo caso, notiamo che la libertà di culto trova nelle normative urbanistiche comunali -che regolano il territorio anche in merito ai luoghi religiosi presenti e futuri- forme di confronto, negoziazione e anche di conflitto.

Per il caso dei filippini si pone una prospettiva differente rispetto al loro riconoscimento, che chiama in causa la Chiesa cattolica locale. Come analizzato in precedenza, a differenza dei sikh, gli immigrati cattolici per trovare uno spazio religioso, ossia una chiesa in uso, entrano in relazione con la diocesi di riferimento. L'insediamento di una comunità etnica richiede infatti un confronto con la pastorale dei migranti, l'ufficio diocesano preposto alla loro cura pastorale, che recepisce la richiesta, la valuta e sonda la possibilità di uno spazio da concedere, inclusa la reperibilità di un "sacerdote etnico" per seguire i fedeli. La possibilità di avere in uso una chiesa è quindi frutto di una negoziazione non scontata.

Inoltre, si pone il problema del rapporto tra i cattolici stranieri che si insediano e le comunità parrocchiali o diocesane. In questo senso, si osserva spesso come i gruppi religiosi etnici vivano solitamente in parallelo rispetto alle parrocchie già costituite (Ambrosini, 2016). Una distanza che, da un lato, deriva dal fatto che gli immigrati ricreano comunità sulla base delle proprie necessità, prima descritte, come la ricerca di un'atmosfera familiare e la possibilità di reperire supporto emozionale, sociale e materiale; dall'altro desiderano però essere protagonisti e non fedeli passivi in contesti in cui la ricezione e il riconoscimento della diversità è ancora un processo in divenire 
nonostante i proclami che arrivano dall'alto. Lo stigma che segue gli immigrati anche all'interno delle parrocchie è un nodo critico nonché una sfida rispetto ad una Chiesa che a Milano, e in Lombardia, di fatto sta diventando sempre più multiculturale. Le forme di accettazione a livello locale sono ancora, in entrambi i casi, un processo non privo di difficoltà.

Infine, per comprendere l'insediamento delle comunità e i processi di integrazione che ne possono derivare, consideriamo ora una prospettiva differente, prendendo in esame il civismo. In generale, l'attivismo e le forme di collaborazione e reciprocità rinsaldate in ambito associativo, nel corso del tempo, possono anche portare allo sviluppo di spirito civico nei confronti della più ampia realtà sociale (Putnam, 1993; 2004). Per i nostri casi studio, come indicatore, consideriamo le iniziative solidali volte e promosse verso l'esterno. Nonostante le difficoltà inerenti al riconoscimento delle comunità a livello locale, abbiamo infatti osservato pratiche di altruismo verso il territorio, soprattutto nel lungo periodo segnato dalla pandemia da Covid-19.

Per esempio, nella Bassa Bergamasca, a Cortenuova, uno dei templi frequentati da molti dei bergamini intervistati ha promosso differenti raccolte fondi al proprio interno, riuscendo in questo modo a mobilitare una solidarietà che è andata oltre $\mathrm{i}$ confini della stessa comunità. Il tempio ha infatti deciso di destinare importanti donazioni a livello locale: all'ospedale, all'Avis regionale e alla protezione civile del comune. Significativi atti solidali in un'area profondamente segnata dalla pandemia.

Nel caso dei filippini, alcune delle comunità milanesi, ma anche quella di Como, hanno promosso raccolte fondi per aiutare alcune organizzazioni di volontariato locali, comprese le Caritas delle diocesi di riferimento; somme non di poco conto per una popolazione tipicamente non abbiente, impegnata nel mandare rimesse per aiutare i familiari in patria, e spesso considerata solo come ricettiva di aiuti piuttosto che donatrice.

Queste iniziative, da un lato, indicano che le comunità nel corso de tempo hanno maturato e sviluppato consapevolezza e considerazione rispetto al territorio in cui si sono insediate. Le azioni solidali sono infatti indicatori di rilievo per comprendere il civismo e per valutare i processi di integrazione a livello locale. Mostrano appartenenza ed empatia verso l'area di insediamento, e verso la popolazione che vi risiede. Dall'altro lato va però notato che le pratiche osservate rientrano in un tipo di civismo di stampo prettamente solidaristico, mentre altre iniziative, di tipo rivendicativo, come reclami in merito alle discriminazioni, partecipazioni a tavoli territoriali, mobilitazioni o interlocuzioni sul piano piano politico, non sono al momento osservabili.

In conclusione, possiamo osservare come l'istituzionalizzazione degli spazi religiosi, in termini di sviluppo, riconoscimento e capacità di 
interazione, sia quindi un processo complesso. Le comunità sono coinvolte a livelli differenti nella realtà sociale circostante. Questo indica come il rapporto con il nuovo pluralismo sia un processo in evoluzione, non privo di difficoltà, ma nuovi -ed imprevisti- scenari stanno maturando.

\section{Conclusione}

Il rapporto tra la formazione di comunità religiose e l'integrazione degli immigrati è un ambito di ricerca solo recentemente esplorato in Europa ed in Italia. Uno dei principali nodi di discussione è se le religioni trapiantate dai migranti costituiscano solo spazi etnici transnazionali, ossia rivolti alla madrepatria, o se invece promuovano anche processi di integrazione nella società ricevente.

Per sviluppare da un punto di vista analitico il tema, l'articolo ha avanzato una prospettiva sociologica, che si intreccia e contribuisce a quella istituzionalista, che appunta l'attenzione sulle normative e i processi di governance del fenomeno. Una linea di ricerca che considera i significati e le pratiche socio-religiose promosse dagli immigrati nei propri luoghi di culto.

Quindi sono state prese in esame due comunità religiose, rappresentative dei tipi di pluralismo che gli immigrati hanno promosso in Italia, e della loro partecipazione al mercato del lavoro italiano. La chiave compartiva ha invece permesso di valutare analogie e differenze in merito ai processi di integrazione.

Dopo aver fornito un quadro di sintesi della presenza dei sikh e dei cattolici filippini in Lombardia, il primo livello di indagine ha analizzato la geografia religiosa, evidenziando come le due comunità, nonostante le difficoltà, tramite due differenti tipi di processi mimetici, abbiano istituito i loro luoghi di culto. Da un lato, rendendo sacri edifici secolari, dall'altro risignificando in chiave etnica chiese in disuso.

Un secondo livello ha invece esaminato i significati e le attività sociali promosse dai fedeli. È così emersa una "controstoria" religiosa delle rispettive esperienze lavorative. In questo senso, la religione è un importante fattore di resilienza, in grado di fornire capitale sociale, benessere emotivo e riconoscimento, riabilitando anche gli immigrati dall'immagine stereotipata e dallo stigma che li segue nell'ambito lavorativo.

Un terzo ambito di ricerca ha preso in esame il riconoscimento di queste comunità a livello locale, mostrando come talvolta i templi siano stati oggetto di contestazione nel territorio di insediamento, mentre la presenza di cattolici stranieri rappresenta invece una sfida multiculturale per le parrocchie italiane locali, in termini di dialogo e riconoscimento. Oltre a queste 
difficoltà, è stata però messa in evidenza anche la promozione da parte dei fedeli immigrati di importanti attività solidali verso la realtà circostante; pratiche civiche che rivelano empatia e consapevolezza nei confronti della popolazione locale in un periodo segnato dalla pandemia.

In conclusione, possiamo osservare come le comunità in esame siano diventate importanti riferimenti socio-relazionali per l'inserimento della popolazione immigrata. In questo senso, se la società ricevente è posta di fronte al tema della presenza di nuove identità religiose, ad uno sguardo analitico, rispetto allo spaesamento che ne può derivare, emergono anche evidenze su come i luoghi di culto rappresentino spazi ove si siano sviluppati elementi e dinamiche utili all'integrazione. Questo implica anche un approccio più flessibile rispetto alla presenza della religione nello spazio pubblico, non solo come fattore divisivo, ma anche come snodo per poter intessere processi di interlocuzione, dialogo e coesione rispetto ad una popolazione che si è progressivamente insediata sul territorio.

\section{Riferimenti bibliografici}

Ambrosini, M. (2020). Sociologia delle Migrazioni, Terza Edizione. Bologna: il Mulino.

Ambrosini, M. (2016). Protected but separate: International immigrants in the Italian Catholic Church. In D. Pasura, M. B. Erdal (eds), Migration, Transnationalism and Catholicism, pp. 317-335. London: Palgrave Macmillan.

Ambrosini, M., Bonizzoni, P., \& Molli, S. D. (2021a) How religion shapes immigrants' integration: The case of Christian migrant churches in Italy. Current Sociology. Doi: 10.1177/0011392120979018.

Ambrosini, M., Bonizzoni, P., \& Molli, S. D. (2021b) Il welfare informale dei migranti. Chiese etniche e processi di inclusione sociale a Milano. Rassegna Italiana di Sociologia 62 (1), pp. 165-196. Doi: 10.1423/100625.

Ambrosini, M., Naso, P., \& Paravati, C. (2018). Il Dio dei migranti: pluralismo, conflitto, integrazione. Bologna: il Mulino.

Berger, P. L., Davie, G., \& Fokas, E. (2010). America religiosa, Europa laica? Perché il secolarismo europeo è un'eccezione. Bologna: il Mulino.

Bertolani, B. (2013). I sikh. In E. Pace (eds), Le religioni nell'Italia che cambia. Mappe e bussole, pp. 31-46, Roma: Carocci.

Bonifacio, G. T., \& Angeles, V. S. M. (2010). Gender, religion, and migration: pathways of integration. Rowman \& Littlefield.

Cadge, W., \& Ecklund, E. H. (2007). Immigration and religion. Annual Review of Sociology, (33), 359-379. Doi: 10.1146/annurev.soc.33.040406.131707.

Caritas-Migrantes, Fondazione (2020). XXIX Rapporto Immigrazione 2020. Conoscere per Comprendere. Todi: Tau Editrice Srl.

Casanova, J. (2007). Immigration and the new religious pluralism: A European 
Union/United States comparison. In, T. Banchoff(Eds.), Democracy and the new religious pluralism, pp. 59-83, Oxford University Press.

Connor, P. (2012). Balm for The Soul: Immigrant Religion and Emotional WellBeing. International Migration, 50(2), 130-157. Doi: 10.1111/j.14682435.2010.00623.x.

Dorais, L. J. (2007). Faith, hope and identity: Religion and the Vietnamese refugees. Refugee Survey Quarterly, 26(2), 57-68. Doi: 10.1093/rsq/hdi0227.

Ebaugh, H. R., \& Chafetz, J. S. (1999). Agents for cultural reproduction and structural change: The ironic role of women in immigrant religious institutions. Social Forces, 78(2), 585-612. Doi: 10.1093/sf/78.2.585.

Ebaugh, H. R., \& Curry, M. (2000). Fictive kin as social capital in new immigrant communities. Sociological Perspectives, 43(2), 189-209. Doi: 10.2307/1389793.

Ferrari, A. (2012). La libertà religiosa in Italia: un percorso incompiuto. Roma: Carocci.

Foley, M. W., \& Hoge, D.R. (2007). Religion and the new immigrants: How faith communities form our newest citizens. Oxford University Press.

Foner, N., \& Alba, R. (2008). Immigrant religion in the US and Western Europe: Bridge or barrier to inclusion? International migration review, 42(2), 360-392. Doi: $10.1111 / j .1747-7379.2008 .00128 . x$.

Gallo, E. (2012). Creating Gurdwaras, Narrating Histories: Perspectives on the Sikh Diaspora in Italy. South Asia Multidisciplinary Academic Journal, 6, Online since 28 December. Doi: 10.4000/samaj.3431.

Gozdziak, E. M. (2002). Spiritual emergency room: the role of spirituality and religion in the resettlement of Kosovar Albanians. Journal of Refugee Studies, 15(2), 136-152.

Guest, K. J. (2003). God in Chinatown: Religion and survival in New York's evolving immigrant community. New York: NYU Press.

Hervieu-Léger, D. (2000). Religion as a Chain of Memory. New Brunswick, NJ: Rutgers University Press.

Hirschman, C. (2004). The role of religion in the origins and adaptation of immigrant groups in the United States. International Migration Review, 38(3), 1206-1233. Doi: 10.1111/j.1747-7379.2004.tb00233.x.

Idos-Confronti (2020). Dossier statistico Immigrazione. Centro Studi e Ricerche IDOS in partenariato con il Centro Studi Confronti, Roma.

INPS (2020). Lavoratori domestici, serie: Statistiche in breve. A cura del Coordinamento Generale Statistico Attuariale. Pubblicato online giugno 2020.

Ismu, Fondazione (2019). Comunicato stampa: Immigrati e religioni in Italia - 23 luglio. https://www.ismu.org/comunicato-stampa-immigrati-e-religioni-in-italia/.

Levitt, P. (2007). God needs no passport. New York: The New.

Ley, D. (2008). The immigrant church as an urban service hub. Urban Studies, 45(10), 2057-2074. Doi: 10.1177/0042098008094873.

Lutz, H. (2011). The new maids: Transnational women and the care economy. Zed Books Ltd.

McMichael, C. (2002). 'Everywhere is Allah's place': Islam and the everyday life of Somali women in Melbourne, Australia. Journal of refugee studies, 15(2), 171-188. Doi: 10.1093/jrs/15.2.171. 
Menjívar, C. (2006). Liminal legality: Salvadoran and Guatemalan immigrants' lives in the United States. American journal of sociology, 111(4), 999-1037. Doi: $10.1086 / 499509$.

Min, P. G. (2010). Preserving ethnicity through religion in America: Korean Protestants and Indian Hindus across generations. NYU Press.

Molli, S. D. (2020). Immigrant Religious Networks in Milan: Ethnic Churches as Source of Social Capital. Mondi Migranti, 1, 97-116. Doi: 10.3280/MM2020001006

Mooney, M. A. (2009). Faith makes us live: Surviving and thriving in the Haitian diaspora. Berkeley: University of California Press.

Nyhagen, L., \& Halsaa, B. (2016). Religion, gender and citizenship: Women of faith, gender equality and feminism. Palgrave Macmillan.

Omenetto, S. (2015). Dio non ha passaporto. I gurdwara dal Punjab all'Italia. Studi e Materiali di Storia delle Religioni, 81(2), 616-650.

Orsi, R. A. (2010). The Madonna of 115th Street: faith and community in Italian Harlem, 1880-1950. Yale University Press. (third edition, first edition published: 1985, second edition: 2002).

Pace, E. (2013). Le religioni nell'Italia che cambia: mappe e bussole. Roma: Carocci.

Pace, E. (2018). Conclusion. Religions, 9(4), 94. Doi: 10.3390/rel9040094.

Putnam, R. D. (1993). La tradizione civica nelle regioni italiane. Trad. it., Milano: Mondadori (ed. or. 1993).

Putnam, R. D. (2004). Capitale sociale e individualismo. Crisi e rinascita della cultura civica in America, trad. it., Bologna: il Mulino (ed. or. 2000).

Ricucci, R. (2017). Diversi dall'Islam. Figli dell'immigrazione e altre fedi. Bologna: il Mulino.

Sanfilippo, M. (2007). Parrocchie ed immigrazione negli Stati Uniti. Studi Emigrazione, 44(168), 993-1005.

Stepick, A., Rey, T., \& Mahler, S. J. (2009). Churches and Charity in the Immigrant City: Religion, Immigration, and Civic Engagement in Miami. Rutgers University Press.

Triandafyllidou, A., \& Magazzini, T. (2020). Governing religious diversity across the world: comparative insights. In, Ibidem (Eds.) Routledge Handbook on the Governance of Religious Diversity, pp. 309-319, Routledge.

Tweed, T. (1997). Our lady of the exile: Diasporic religion at a Cuban Catholic shrine in Miami. New York: Oxford University Press.

Vecoli, R. J. (1969). Prelates and peasants: Italian immigrants and the Catholic church. Journal of Social history, 2(3), 217-268.

Warner, R. S. (2000). Religion and new (post-1965) immigrants: Some principles drawn from field research. American Studies, 41(2/3), 267-286.

Yang, F., \& Ebaugh, H. R. (2001), Transformations in new immigrant religions and their global implications. American Sociological Review, 66(2), 269-288. Doi: $10.2307 / 2657418$. 